Johnson and Dr. Th. Gill (NA'TuRE, vol. xxvi. pp. 453, 574) to both of whom a reply is due, and should bave been given sooner had I not been absent from Florence and otherwise engaged.

Firstly, I must correct my assertion as to the occurrence of Malacocephalus lavis in the Mediterranean; after having examined the type specimen and that mentioned by Mr. Johnson, both in the British Museum and after a further examination of my specimens, which I had considered as young Malaco:ephati, I have now not the slightest doubt that they are quite distinct. They are an undescribed and most interesting form of Macrurids allied to Coryphenoides, which I propose calling Hymenocephalus italicus. I have in my possession six specimens, both adult and young; in two of the former I have found the ovaries fully deve. loped with mature ova.

As to the "singular fish of a deep black colour with small eyes, a naked skin, and a most abyssal physiognomy," which I got at Messina, it has no connection whatever with Chiasmodon niger, but is, as I before assserted, a Stomiatid, very differen from all the known forms, including Dr. Günther's Bathyothis. It stands apart in many lespects, and is the type of a new genus and perhaps of a new section of that singular family. I intend shortly to describe and figure it under the name of Bathophilus nigerrimus, along with other strange fish collected during my deep-sea and ichthyological researches in the Mediterranean.

Henry Hillyer Gigi.ioli

R. Zoological Museum, Florence, December I 7

\section{Electrical Phenomenon}

ON retiring to bed shortly after midnight on the $I 3^{\text {th }}$ inst., I experienced a phenomenon which, though not of itself uncom mon, was, I think, unusually developed. On pulling off a flannel vest which I wear next my skin, over my head, I became conscious of a strange sensation in the, a.ter, accompanied by a distinct crackling noise, and bright sparks which vere plainly visible in the dimly lighted room. To make sure that I was not the subject of a delusion, I repeated the operation many times, in each case rubbing the flannel half-a-dozen times-not more-against my hair. Not only were the same phenomena observable every time, but also if, after removing the flannel I then approached my knuckles to that part of it which had been in contact with the hair, a whole volley of sparks passed between the flannel and each knuckle at a distance of not less than two inches. As often as I repeated the experiment, so often did the phenomena repeat themselves, until I at lenoth retired to bed not altogether without apprehension, that I might awake in the night with the bed-clothes on fire, by reason of the discharge of some extra big spark between my bair and a convenient blanket. No such catastrophe, however, occurred, and on repeating the operations the next morning, I could not reproduce the phenomena. The next evening 1 again repeated the experiment, and this time by very violent rubbing could just get a faint discharge between the flannel and knuckles when almost in contact. On other nights since these I have not succe eded in getting any such effect, or at most a very feeble one. To what, then, am I to attribute the marked difference of the first night? Was it due to something peculiar in the condition of the bair, the air, or the flannel? Perhaps some of your readers can suggest. As regards the first of these $I$ ought to state that it had, on the afternoon of the I 3 th, been subjected to the operations of cutting, shampooing, and brushing "by machinery," at the hand; of the barber. That was, however, seven hours earlier in the day, and any electricity developed by the friction of the last operation ought to have been dissipated long before twelve o'clock-especially as the night was damp and misty.

29, Victoria Road, Finsbury Park, December I9

A. J. K.

\section{PHOTOGRAPHING THE CORONA}

PROBLEMS of the highest interest in the physics of our sun are connected, doubtless, with the varying forms which the coronal light is known to assume, but these would seem to admit of solution only on the condition of its being possible to study the corona continuously,

I "On a method of Photographing the Solar Corcna without an Eclipse." Paper read at the Royal Society by William Huggins, D.C.L., LL.D., P.R.S., December $2 \mathrm{I}$. and so to be able to confront its changes with the other variable phenomena which the sun presents. "Unless some means be found," says Prof. C. A. Young, "for bringing out the structures round the sun which are hidden by the glare of our atmosphere, the progress of our knowledge must be very slow, for the corona is visible only about eight days in a century, in the aggregate, and then only over narrow stripes on the earth's surface, and but from one to five minutes at a time by any one observer" ("The Sun," p. 239).

The spectroscopic method of viewing the solar prominences fails, because a large part of the coronal light gives a continuous spectrum. The successful photograph of the spectrum of corona taken in Egypt, with an instrument provided with a slit, under the superintendence of Prof. Schuster during the solar eclipse of May 17, I882, shows that the coronal light as a whole, that is the part which gives a continuous spectrum, as well as the other part of the light which may be resolved into bright lines, is very strong in the region of the spectrum extending from about $\mathrm{G}$ to $\mathrm{H}$. It appeared to me, therefore, very probable that by making exclusive use of this portion of the spectrum it might be possible under certain conditions, about to be described, to photograph the corona without an eclipse.

In the years I 866-68 I tried screens of coloured glasses and other absorptive media, by which I was able to insolate certain portions of the spectrum with the hope of seeing directly, without the use of the prism, the solar prominences (Monthly Notices, vol. xxviii. p. 88, and vol. xxix. p. 4). I was unsuccessful, for the reason that I was not able by any glasses or other media to isolate so very restricted a portion of the spectrum as is represented by a bright line. This cause of unsuitableness of this method for the prominences which give bright lines only, recommends it as very promising for the corona. If by screens of coloured glass or other absorptive media the region of the spectrum between $\mathrm{G}$ and $\mathrm{H}$ could be isolated, then the coronal light which is here very strong would have to contend only with a similar range of refrangibility of the light scattered from the terrestrial atmosphere. It appeared to me by no means improbable that under these conditions the corona would be able so far to hold its own against the atmospheric glare, that the parts of the sky immediately about the sun where the corona was present would be in a sensible degree brighter than the adjoining parts where the atmospheric light alone was present. It was obvious, however, that in our climate and low down on the earth's surface, even with the aid of suitable screens, the addition of the coronal light behind would be able to increase, but in a very small degree, the illumination of the sky at those places where it was present. There was also a serious drawback from the circumstance that although this region of the spectrum falls just within the range of vision, the sensitiveness of the eye for very small differences of illumination in this region near its limit of power is much less than in more favourable parts of the spectrum, at least such is the case with my own eyes. There was also another consideration of importance, the corona is an object of very complex form, and full of details depending on small differences of illumination, so that even if it could be glimpsed by the eye, it could scarcely be expected that observations of a sufficiently precise character could be made to permit of the detection of the more ordinary changes which are doubtlessly taking place in it.

These considerations induced me not to attempt eyeobservations, but from the first to use photography, which possesses extreme sensitiveness in the discrimination of minute differences of jllumination, and also the enormous advantage of furnishing a permanent record from an instantaneous exposure of the most complex forms. I have satisfied myself by some laboratory experiments that under suitable conditions of exposure and development a photographic plate can be made to record minute differ- 
ences of illumination existing in different parts of a bright object, such as a sheet of drawing paper, which are so subtle as to be at the very limit of the power of recognition of a trained eye, and even, as it appeared to me, those which surpass that limit.

My first attempts at photographing the corona were made with photographic lenses, but uncertainty as to the state of correction of their chromatic aberration for this part of the spectrum, as well as some other probable sources of error which I wished to avoid, led me to make use of a reflecting telescope of the Newtonian form. The telescope is by Short, with speculum of 6 inches diameter, and about $3 \frac{1}{2}$ feet focal length. A small photographic camera was fastened on the side of the telescope tube, and the image of the sun after reflection by the small plane speculum was brought to focus on the ground glass. The absorptive media were placed immediately in front of the sensitive film, as in that position they would produce the least optical disturbance. Before the end of the telescope was fixed a shutter of adjustable rapidity which reduced the aperture to 2 inches. This was connected with the telescope tube by a short tube of black velvet for the purpose of preventing vibrations from the moving shutter reaching the telescope. On account of the shortness of the exposures it was not necessary to give motion to the telescope.

It was now necessary to find an absorptive medium which would limit the light received by the plate to the portion of the spectrum from about $\mathrm{G}$ to $\mathrm{H}$. There is a violet (pot) glass made, which practically does this. I had a number of pieces of this glass ground and polished on the surfaces. Three or four of these could be used together, castor-oil being placed between the pieces to diminish the reflection of light at their surfaces. Some inconvenience was found from small imperfections within the glass, and it would be desirable in any future experiments to have a larger supply of this glass, from which more perfect pieces might be selected.

In my later experiments I used a strong and newly made solution of potassic permanganate, in a glass cell with carefully polished sides. This may be considered as restricting the light to the desired range of wave-length, since light transmitted by this substance in the less refrangible parts of the spectrum does not affect the photographic plates.

Different times of exposure were given, from so short an exposure that the sun itself was rightly exposed, to much more prolonged exposures, in which not only the sun itself was photographically reversed, but also the part of the plates extending for a little distance from the sun's limb.

Gelatine plates were used, which were backed with a solution of asphaltum in benzole.

After some trials I satisfied myself that an appearance peculiarly coronal in its outline and character was to be seen in all the plates. I was, however, very desirous of trying some modifications of the methods described, with the hope of obtaining a photographic image of the corona of greater distinctness, in consequence of being in more marked contrast with the atmospheric illumination.

Our climate is very unpropitious for such observations, as very few intervals, even of short duration, occur in which the atmospheric glare immediately about the sun is not very great. Under these circumstances I think it is advisable to describe the results I have obtained, without further delay.

The investigation was commenced at the end of May of this year, and the photographs were obtained between June and September 28.

The plates which were successful are twenty in number. In all these the coronal form appears to be present. This appearance does not consist simply of increased photographic action immediately about the sun, but of distinct coronal forms and rays admitting in the best plates of measurement and drawing from them. This agreement in plates taken on different days with different absorptive media interposed, and with the sun in different parts of the field, together with other necessary precautions observed, makes it evident that we have not to do with any instrumental effect.

The plates taken with very short exposures show the inner corona only, but its outline can be distinctly traced when the plates are examined under suitable illumination. When the exposure was increased, the inner corona is lost in the outer corona, which shows the distinctly curved rays and rifts peculiar to it.

In the plates which were exposed for a longer time, not only the sun but the corona also is photographically reversed, and in these plates, having the appearance of a positive, the white reversed portion of the corona is more readily distinguished and followed in its irregularly sinuous outline than is the case in those plates where the sun only is reversed, and the corona appears, as in a negative, dark.

Prof. Stokes was kind enough to allow me to send the originals to Cambridge for his examination, and I have his permission to give the following words from a letter I received from him: "The appearance is certainly very corona-like, and I am disposed to think it probable that it is really due to the corona."

Prof. Stokes' opinion was formed from the appearance on the plates alone, without any knowledge of their orientation, and without the means of comparing them with the eclipse plates taken on May 17.

I have since been allowed, through the kindness of Capt. Abney, to compare my plates with those taken of the corona in Egypt during the eclipse of May last. Though the corona is undergoing doubtless continual changes, there is reason to believe that the main features would not have suffered much alteration between May 17 and September 28, when the last of my plates was taken. This comparison seems to leave no doubt that the object photographed on my plate is the corona. The more prominent features of the outer corona correspond in form and general orientation, and the inner corona, which is more uniform in height and definite in outline, is also very similar in my plates to its appearance in those taken during the eclipse.

Measures of the average height of the outer and of the inner corona in relation to the diameter of the sun's image are the same in the eclipse plates as they are in my plates taken here.

There remains little doubt that by the method described in this paper, under better conditions of climate, and especially at considerable elevations, the corona may be successfully photographed from day to day with a definiteness which would allow of the study of the changes which are doubtlessly always going on in it. By an adjustment of the times of exposure, the inner or the outer corona could be obtained as might be desired. It may be that by a somewhat greater restriction of the range of refrangibility of the light which is allowed to reach the plate, a still better result may be obtained.

Plates might be prepared sensitive to a limited range of light, but the rapid falling oft of the coronal light about $\mathrm{H}$ would make it undesirable to endeavour to do without an absorptive screen. Lenses properly corrected might be employed, but my experience shows that excessive caution would have to be taken in respect of absolute cleanness of the surfaces and of some other points. There might be some advantage in intercepting the direct light of the sun itself by placing an opaque disk of the size of the sun's image upon the front surface of the absorptive screen. Though for the reasons I have already stated I did not attempt eye-observations, there seems no reason why, with suitable screens and under suitable atmospheric conditions, the corona should not be studied directly by the eye. There might be some 
advantages in supplementing the photographic records by direct eye-observations. I regret that the very few occasions on which it has been possible to observe the sun has put it out of my power to make further experiments in these and some other obvious directions.

P.S. - [I have Capt. Abney's permission to add the following letter this day received from him ;-" A careful examination of your series of sun-photographs, taken with absorbing media, convinces me that your claim to having secured photographs of the corona with an uneclipsed sun is fully established. A comparison of your photographs with those obtained during the eclipse which took place "in May last, shows not only that the general features are the same, but also that details, such as rifts and streamers, have the same position and form. If in your case, the coronal appearances be due to instrumental causes, I take it that the eclipse photographs are equally untrustworthy, and that my lens and your reflector have the same optical defects. I think that evidence by means of photography of the existence of a corona at all is as clearly shown in the one case as in the other."December I 5, I882.]

\section{A WEDGE AND DIAPHRAGM PHOTOMETER}

A NEW photometer, shown in perspective in the figure, A has lately been constructed by Mr. Sabine. The stand supports a straight horizontal tube, at one end of which is a paraffin lamp, and at the other an eyepiece. The middle portion of the tube is cut away, and has, slipped over it, a collar to which a frame is attached, carrying a wedge of neutral-tinted glass, adjustable by means of a rack and pinion. Inside the collar is fixed a transverse disc of ground opal glass, which the paraffin lamp illuminates to a definite degree. This disc constitutes the field of comparison, the illumination of which is

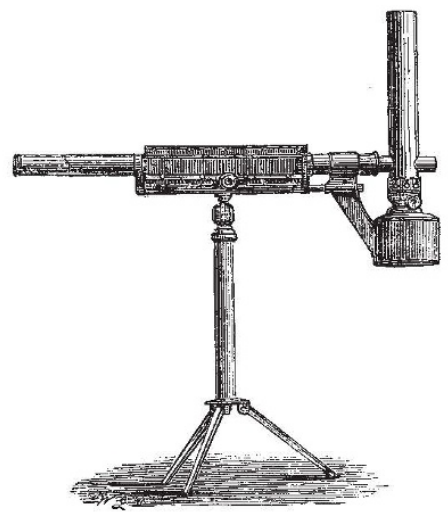

adjustable by means of a series of diaphragms of known aperture at the end near to the paraffin lamp. At the side, between the wedge and the collar which carries it, is a narrow pane of ground opal glass, just behind which a small mirror is fixed at an angle of $45^{\circ}$ to the axis of the tube. This mirror is supported from the centre of the transverse opal disc in such a way, that the support is hidden from the observer by the mirror itself, an arrangement which insures the apparent juxtaposition of the illuminated surfaces which have to be compared. The light to be measured is placed on the right-hand side of the photometer; and the collar is turned so that the light falls normally upon the face of the wedge, passes through the wedge, through the pane of opal glass, and is incident upon the mirror, which reflects a portion of it to the eye of the observer. The wedge is then shifted, if necessary, to interpose a greater or less thickness of absorbing medium, until a balance is obtained, that is until the apparent illumination of the mirror is equal to that of the field of comparison, in the midile of which it is seen. If the range of the wedge is insufficient to admit of this, the degree of illumination of the field is altered, by means of the diaphragms, and the wedge is then adjusted.

The employment of glass wedges for photometric comparisons is not new, having been already used by both Xavier de Maistre and Quetelet; but no practical photometer based upon this method has hitherto been constructed. The employment of diaphragms for extending the range of the wedge is found to work well and to enable the operator to adjust the illumination of the field with exactitude, the bright part of the paraffin flame being of course, kept opposite to, and so as to well cover the diaphragm aperture. A table is constructed giving for each position of the wedge and for each diaphragm, the value, in standard candles, of any light placed at a distance of one metre from the instrument; and if the light be placed at any other distance, the number in the table has simply to be multiplied by the square of the actual distance in metres. For ascertaining approximately the amount of light which passes through any given coloured glass, for example, orange glass, the eyepiece is furnished with a rotary disc containing small panes of white and different coloured glasses, either of which can be interposed at pleasure.

This photometer is being made by Messrs. Elliott Bros., in two forms, one for use as a portable photometer, as shown in the figure, and the other on a more solid stand, for laboratory purposes.

ON THE OCCURRENCE OF GREAT TIDES SINCE THE COMMENCEMENT OF THE GEOLOGICAL EPOCH ${ }^{\mathrm{I}}$

$\mathrm{I} \mathrm{T}$ will I daresay be within the recollection of many of those who are now present that I was honoured by the invitation to deliver the opening lecture in this hall last year. In response to that invitation I addressed to you a discourse which I ventured to call "A Glimpse through the Corridors of Time." Accounts of it have appeared in very many quarters, both at home and abroad. I am myself responsible for the account which appeared in the columns of NATURE, as well as for the pamphlet form in which the lecture has since been issued. The chief reason why I now recur to the subject remains to be stated. Among the various comments which have been made upon that address, some are by no means favourable to the views I ventured to put forward, and they have been the theme of considerable discussion. $\mathrm{Up}$ to the present I have not made any reply to the criticisms which have appeared ; I postponed doing so until a suitable opportunity should have arisen for a review of the whole subject. Your kindness in inviting me once again to address this great Institute has afforded such an opportunity, and with your permission I propose to preface the subject of my lecture this evening by a reply to those critics who have honoured me with their attention.

Let me recall to you very briefly the subject of that lecture, so as to enunciate clearly the point as to which an issue has been raised. You will perhaps recollect that the lecture treated principally of the tidal relations between the earth and the moon, of the influence of the tides during ages past, and of the future which awaits the earth-moon system during ages to come. I pointed out that at the present moment the orbit of the moon must be gradually growing in size, that this gradual increase of the distance from the earth to the moon is essentially non-periodic, and thus is totally different to the ordinary lunar irregularities which are recognisted in rigid-body astronomy. As a consequence of this incessant growth in the moon's distance we see that in past ages the moon must have been appreciably nearer to the earth than it is

I Extract from a lecture delivered at the Midland Institute, Birmi:gham, on November 20, 1882, by Prof. Robert S. Ball, LL.D., F.R.S. Conmunicated by the Author. 